\section{SCIENCE CHINA \\ Physics, Mechanics \& Astronomy}

October 2021 Vol. 64 No. 10: 100331

https://doi.org/10.1007/s11433-021-1758-0

\title{
Quantum enhanced convolutional neural networks for NISQ computers
}

\author{
Dong-Ling Deng ${ }^{1,2 *}$ \\ ${ }^{1}$ Center for Quantum Information, Institute for Interdisciplinary Information Science, Tsinghua University, Beijing 100084, China; \\ ${ }^{2}$ Shanghai Qi Zhi Institute, Shanghai 200232, China
}

Received July 30, 2021; accepted August 3, 2021; published online August 24, 2021

Citation: D.-L. Deng, Quantum enhanced convolutional neural networks for NISQ computers, Sci. China-Phys. Mech. Astron. 64, 100331 (2021), https://doi. org/10.1007/s11433-021-1758-0

The interplay between quantum physics and machine learning may lead to unprecedented perspectives for both fields [1]. On the one hand, ideas and techniques from machine learning, or more broadly artificial intelligence, can be exploited to tackle challenging problems in the quantum domain. On the other hand, judiciously designed quantum algorithms may also enhance, speed up, or innovate machine learning with quantum computers. Undoubtedly, the studies of the interplay between quantum physics and machine learning would benefit both fields, and the emergent research frontier of quantum machine learning has become one of today's most rapidly growing interdisciplinary fields [1].

In ref. [2], the authors proposed hybrid quantum-classical convolutional neural networks (QCCNNs), with an architecture suitable for noisy intermediate scale quantum (NISQ) devices [3]. Unlike other quantum convolutional neural networks, such as those proposed in refs. [4,5], QCCNNs integrate parametric quantum circuits into the classical convolutional neural network architecture by replacing the classical feature map in the convolutional layer with a "quantum feature map", which is essentially a variational quantum circuit and maps the small windows of the input data into a scalar. More specifically, the quantum feature map first encodes the data inside each window into a quantum state, then applies a variational quantum circuit to this quantum state and measures the expectation value of a certain global quantum operator to output a single scalar for the

*Corresponding author (email: dldeng@tsinghua.edu.cn) next layer. Since the window sizes of convolutional neural networks are often selected to be relatively small, the QCCNN approach merits an intriguing advantage of exploiting qubit encoding while maintaining using only a small number of qubits. This enables QCCNNs to circumvent the reliance upon a quantum random access memory, which is resource demanding, to load classical data or prepare initial input quantum states.

This work may inspire a number of future studies. First, it would be interesting and important to study whether QCCNNs suffer from the barren plateau (i.e., vanishing gradient) problem. This is a crucial problem in training various quantum variational circuits. In addition, quantum adversarial machine learning has attracted tremendous attention recently [6]. It would be interesting and of practical importance to study the robustness of QCCNNs to adversarial perturbations and develop effective defense strategies. Finally, an experimental demonstration of QCNNs should also mark a notable step toward practical applications of NISQ devices in machine learning.

1 S. Das Sarma, D. L. Deng, and L. M. Duan, Phys. Today 72, 48 (2019), arXiv: 1903.03516.

2 J. Liu, K. H. Lim, K. L. Wood, W. Huang, C. Guo, and H.-L. Huang, Sci. China-Phys. Mech. Astron. 64, 290311 (2021).

3 J. Preskill, Quantum 2, 79 (2018).

4 I. Cong, S. Choi, and M. D. Lukin, Nat. Phys. 15, 1273 (2019), arXiv: 1810.03787.

5 S. J. Wei, Y. H. Chen, Z. R. Zhou, and G. L. Long, arXiv: 2104.06918.

6 S. Lu, L. M. Duan, and D. L. Deng, Phys. Rev. Res. 2, 033212 (2020), arXiv: 2001.00030. 